\title{
Endosperm and seed transcriptomes reveal possible roles for small RNA pathways in wild tomato hybrid seed failure
}

Ana Marcela Florez-Rueda ${ }^{1,2 *}$, Flurin Fiscalini ${ }^{1}$, Morgane Roth ${ }^{1,3}$, Ueli Grossniklaus ${ }^{1}$, and Thomas Städler ${ }^{2}$

${ }^{1}$ Department of Plant and Microbial Biology \& Zurich-Basel Plant Science Center, University of Zurich, 8008 Zurich, Switzerland

${ }^{2}$ Institute of Integrative Biology \& Zurich-Basel Plant Science Center, ETH Zurich, 8092 Zurich, Switzerland

${ }^{3}$ Current address: Agroscope, Research Division Plant Breeding, 8820 Wädenswil, Switzerland

*Author for Correspondence: Ana Marcela Florez-Rueda, Department of Plant and Microbial Biology \& Zurich-Basel Plant Science Center, University of Zurich, 8008 Zurich, Switzerland. Tel: +41 4463 48251; Fax: +41 4463 48204; E-mail: anamflorezrueda@gmail.com 


\section{Abstract}

2 Crosses between the wild tomato species Solanum peruvianum and S. chilense result in hybrid seed

3 failure (HSF), characterized by endosperm misdevelopment and embryo arrest. We previously showed

4 that genomic imprinting, the parent-of-origin-dependent expression of alleles, is perturbed in hybrid

5 endosperm, with many of the normally paternally expressed genes losing their imprinted status. Here,

6 we report transcriptome-based analyses of gene and small RNA expression levels. We identified 2,295

7 genes and 468 small RNAs (sRNAs) as differentially expressed (DE) when comparing reciprocal hybrid

8 seed to seeds and endosperms from the two within-species crosses. Our analyses uncovered a pattern of

9 overdominance in endosperm gene expression in both cross directions, in marked contrast to the patterns

10 of sRNA expression in whole seeds. Intriguingly, patterns of increased gene expression resembled the

11 previously reported increased maternal expression proportions in hybrid endosperms. We identified

12 physical clusters of sRNAs; DE sRNAs exhibited reduced levels of expression in hybrid seeds from both

13 cross directions. Moreover, sRNAs mapped to genes coding for key proteins involved in epigenetic

14 regulation of gene expression, suggesting a regulatory feedback mechanism. We describe examples of

15 genes that are targets of sRNA-mediated gene silencing; in these cases, reduced sRNA expression was

16 concomitant with increased gene expression in hybrid seeds. Our analyses also show that S. peruvianum

17 dominance impacts gene and sRNA expression in hybrid seeds. Overall, our study indicates roles for

18 sRNA-mediated epigenetic regulation in HSF between closely related wild tomato species.

19

20 Key words: postzygotic isolation, small RNAs, seed development, epigenetic, transcriptomics,

21 Solanum. 


\section{Introduction}

23 The establishment of reproductive barriers between diverging lineages is a basic component of the

24 speciation process and thus of major interest in evolutionary biology (Coyne and Orr 2004). In this

25 study, we assess the molecular correlates of hybrid seed failure (HSF), a form of postzygotic barrier

26 acting early in seed development of many flowering plants. In the angiosperm seed, embryo and

27 endosperm are the products of two independent fertilization events. The endosperm is usually a triploid

28 tissue that nourishes the growing embryo; failure of proper endosperm development often leads to

29 embryo arrest and is considered the main cause of HSF (Rebernig et al. 2015; Garner et al. 2016; Oneal

30 et al. 2016). HSF has been frequently observed upon hybridization of closely related homoploid plant

31 species as well as between lineages differing in ploidy (Beamish 1955; Rick 1963; Scott et al. 1998;

32 Dilkes et al. 2008; Jullien and Berger 2010; Lu et al. 2012; Burkart-Waco et al. 2013; Rebernig et al.

33 2015; Oneal et al. 2016).

34 From an evolutionary perspective, the developing seed can be viewed as an arena in which the two

35 parental genomes 'collide'. Any differences in parental optima for resource allocation to progeny

36 (representing parental conflict) are expected to manifest in the endosperm (Haig and Westoby 1991;

37 Haig 2013). The ratio of 'effective' parental genomic contributions in the endosperm appears to largely

38 determine the success or failure of particular crosses, an interpretation bolstered by the frequent

39 observation that postzygotic barriers can be weakened by manipulating the ploidy of one of the parents

40 (Johnston et al. 1980; Josefsson et al. 2006; Lafon-Placette and Köhler 2016). Transgressive and

41 complementary hybrid seed phenotypes are common and thought to reveal different levels of parental

42 conflict between lineages (Lu et al. 2012; Haig 2013; Rebernig et al. 2015; Florez-Rueda, Paris, et al.

43 2016). These observations have led to the hypothesis that parent-of-origin-dependent allelic expression

44 (i.e. genomic imprinting) might be causally involved in HSF. Genomic imprinting is an epigenetic

45 phenomenon causing the preferential expression of alleles depending on their parental origin. In

46 flowering plants, while occurring also in the embryo, genomic imprinting is prevalent in the endosperm

47 and critical for proper seed development (Berger 2003).

48 Although perturbed genomic imprinting has been shown to be a molecular correlate of HSF

49 (Gutierrez-Marcos et al. 2003; Josefsson et al. 2006; Walia et al. 2009; Jullien and Berger 2010; Wolff

50 et al. 2015; Florez-Rueda, Paris, et al. 2016; Lafon-Placette et al. 2018), successful seed development 
51 results from the precise orchestration of additional genomic and developmental processes. Other

52 molecular processes during seed formation, like de-repression of transposable elements (TEs; Fultz et al.

53 2015; Martínez and Köhler 2017) and gene regulation mediated by small RNAs (sRNAs; Bourc'his and

54 Voinnet 2010; Ng et al. 2012; Lu et al. 2012) likely act in the endosperm to determine the success or

55 failure of particular cross combinations. Of particular interest are sRNAs; these RNA forms are involved

56 in plant development, reproduction, and genome reprogramming (Haig 2013; Benkovics and

57 Timmermans 2014; Borges and Martienssen 2015; Martínez and Köhler 2017).

58 For instance, microRNAs (miRNAs) are post-transcriptional regulators of gene expression, and

59 various other types of sRNAs are involved in post-transcriptional gene silencing (PTGS) via transcript

60 cleavage or translational repression as well as in transcriptional gene silencing (TGS), the latter mostly

61 via RNA-directed DNA methylation (RdDM; Matzke and Mosher 2014; Pikaard and Mittelsten Scheid

62 2014; Borges and Martienssen 2015; Cuerda-Gil and Slotkin 2016; D’Ario et al. 2017). Several recent

63 studies point to a pivotal role for sRNA-mediated gene silencing in regulating proper seed development

64 and/or hybrid fitness (Groszmann et al. 2011; Lu et al. 2012; Rodrigues et al. 2013; Vu et al. 2013;

65 Martínez et al. 2016, 2018; Borges et al. 2018). Although our current knowledge regarding sRNA

66 biogenesis and regulatory mechanisms stem mainly from work in the model species Arabidopsis

67 thaliana, it is expected that the underlying concepts apply to most angiosperms, albeit some deviations

68 from the canonical mechanisms may occur in more distantly related taxa, such as our model system

69 Solanum.

70 In this study, we quantified the expression patterns of sRNAs in reciprocal crosses between two

71 wild tomato species that show near-complete HSF, an important postzygotic barrier to interbreeding

72 among several species of wild tomatoes (Solanum section Lycopersicon). Classical studies found high

73 proportions of HSF in reciprocal crosses between the closely related S. peruvianum $(\mathrm{P})$ and $S$. chilense

74 (C) (Rick and Lamm 1955). Following this pioneering work, we have quantified various degrees of seed

75 inviability in reciprocal hybrid crosses involving several species of wild tomatoes. Moreover, we

76 observed differences in the cellular architecture and histology of failing endosperms, as well as strong

77 differences in seed size depending on the direction of hybrid crosses (Roth, Florez-Rueda, Griesser, et

78 al. 2018). Similar HSF-associated phenotypes have been described in different Solanum species and

79 other angiosperm taxa, including interploidy and homoploid hybrid crosses in model species and 
80 important crops (Cooper and Brink 1945; Beamish 1955; Rick 1963; Ortiz and Ehlenfeldt 1992; Scott et

81 al. 1998; Dilkes et al. 2008; Jullien and Berger 2010; Ishikawa et al. 2011; Burkart-Waco et al. 2013;

82 Rebernig et al. 2015).

We previously studied the molecular correlates of HSF in the S. peruvianum-S. chilense case and

84 found that genomic imprinting in the endosperm is systematically perturbed (Florez-Rueda, Paris, et al. 2016), but we did not assess changes in overall expression levels. This intriguing pattern motivated us to investigate the likely epigenetic basis of strong HSF as observed in $S$. peruvianum-S. chilense crosses, with a focus on the possible roles of sRNAs. In the present study, we integrate gene and sRNA expression estimates and study their expression profiles in both normally developing and failing hybrid endosperm and seeds, respectively. We examine the sRNAs' targets and provide examples of representative genes exhibiting changes in gene expression concomitant with sRNA expression variation. By comparing the expression of hybrids and their parents, we further test how expression inheritance patterns are shaped by different 'effective ploidies' of the parental lineages.

\section{Materials and Methods}

97 (http://tgrc.ucdavis.edu, last accessed 16 June 2016). For $S$. peruvianum, we used seeds from accession LA1616 (Dept. Lima, Peru) and for S. chilense, we used seeds from accession LA4329 (Region three different parental combinations: the within-species S. peruvianum case (PP) with plants 1616A and

$1011616 \mathrm{~J}$ as parents, the within-species $S$. chilense (CC) case with plants 4329B and 4329K as parents, and 102 the hybrid cases (PC and CP) with plants 1616A and 4329B in both parental roles in reciprocal crosses.

103 The parental plants were grown from seeds and transferred to a climate chamber before the onset of the

104 experiments. The conditions in the climate chamber were $12 \mathrm{~h}$ light $(18 \mathrm{klux})$ at $22^{\circ} \mathrm{C}$ with $50 \%$ relative 105 humidity and $12 \mathrm{~h}$ darkness $(0 \mathrm{klux})$ at $18^{\circ} \mathrm{C}$ with $60 \%$ relative humidity. For each of the three cross 106 types, hand pollinations were performed and developing fruits were collected on each plant for each 107 cross type. 

own histological analyses (Roth, Florez-Rueda, Griesser, et al. 2018), we chose an early globular

110 embryo stage to collect the material for library preparation. We collected fruits 14 days after pollination

111 (DAP), always in the late afternoon. This developmental stage was chosen because it was early enough

112 to distinguish the developing embryo from the surrounding endosperm tissue, while the latter was large

113 enough to extract RNA in the quantities needed for library preparation. For each plant and cross type,

114 two separate RNA libraries were prepared from endosperm tissue, for a total of 12 endosperm libraries.

115 The raw data for the endosperm transcriptomes has been published before, and detailed methodology for

116 its production was described in Florez-Rueda, Paris, et al. (2016). In brief, fruits were harvested, fixed,

117 and endosperms were laser captured with the Laser-Assisted Microdissection (LAM) technique outlined

118 in Florez-Rueda, Grossniklaus, et al. (2016).

119 The same crossing design described above for endosperm transcriptomes was implemented for the 120 whole-seed sRNA data sets. As we were interested in overall—rather than parent-specific — sRNA

121 expression levels and sRNAs were found to be abundant in all three Arabidopsis seed compartments

122 (Erdmann et al. 2017; Kirkbride et al. 2019), we extracted sRNAs from whole seeds. Moreover, we

123 generated sRNA libraries only from hybrid and normal seeds from plants 1616A and 4329B, serving as

124 parents in both intra- and inter-specific crosses (supplementary figure S1, Supplementary Material

125 online). For these sRNA libraries, we used three replicates for our analyses, each replicate reflecting

126 independent hand-pollination events performed on different days. As for the endosperm transcriptomes,

127 developing fruits were collected at 14 DAP in the late afternoon and immediately placed into RNAlater

128 solution. The samples were immediately transferred to a refrigerator and remained in the RNAlater

129 solution for a minimum of $24 \mathrm{~h}$ and a maximum of $48 \mathrm{~h}$. Whole seeds were dissected in RNase-free

130 water and subjected to consecutive water washes to remove the fruit flesh debris. We collected a

131 minimum of $1 \mu \mathrm{g}$ of seeds from tens of fruits from each cross type and proceeded to sRNA extraction.

132 RNA was extracted using the miRVana RNA isolation kit (Ambion, Life Technologies Corporation,

133 Foster City, CA, USA). sRNA libraries were prepared using the NEXTflex SRNAs-Seq Kit v2

134 according to the manufacturer's protocol (Bioo Scientific Corporation, Austin, TX, USA). Libraries

135 were sequenced in single-end fashion on one lane of an Illumina HiSeq 4000 at the Functional

136 Genomics Center Zurich (www.fgcz.ch). 
139 Mapping of sRNA reads was performed using STAR (Dobin et al. 2013) with a maximum of two 140 mismatches to the SL2.50 assembly of the cultivated tomato reference genome (The Tomato Genome 141 Consortium 2012) deposited in ensemble genomes (https://plants.ensembl.org/

142 Solanum_lycopersicum/Info/Annotation/\#genebuild, last accessed 7 February, 2017). After using 143 Shortstack (Axtell 2013), 122,398 sRNA clusters were identified with default options. Of these, 57,711

144 fell within the coordinates of a gene coding region or its $2.5 \mathrm{~kb}$ flanking regions using BEDTools 145 window command (Quinlan and Hall 2010); these clusters were used for further analyses. Of these 14657,711 clusters and based on the corresponding SL2.50 ensemble annotation of the genome, we 147 annotated 7,112 sRNAs as miRNAs; Shortstack inferred 44 additional miRNAs for a total number of 1487,156 miRNAs. Other forms of non-coding RNAs that were represented in our sRNA libraries included 1492,949 antisense RNAs, rRNAs, tRNAs, snoRNAs, snRNAs, and SRPRNAs. These latter forms were 150 removed before performing differential expression analyses. By using the counts provided by 151 Shortstack, we performed differential gene expression analyses using DESeq2 (Love et al. 2014) in the 152 same manner as for the endosperm transcriptomes (see below).

153 We reanalyzed the endosperm transcriptome data previously produced (Florez-Rueda, Paris, et al. 154 2016). Raw reads were mapped to the SL2.50 assembly of the tomato genome deposited in ensemble 155 genomes (https://plants.ensembl.org/Solanum_lycopersicum/Info/ Annotation/\#genebuild). The tuxedo 156 pipeline (Trapnell et al. 2012) was used for the assembly of reads, mapping to the tomato reference 157 genome, and count estimation. Raw count tables were produced with additional packages of the Tuxedo 158 pipeline, cuffquant and cuffnorm; unnormalized counts per transcript were used for subsequent analyses. 159 Differential gene expression analyses for transcripts as well as for sRNA clusters were performed using 160 DESeq2 (Love et al. 2014), as implemented in the RNAseqWrapper package (Schmid 2017) in R (R

161 Development Core Team 2014). To test for differential gene expression between viable and hybrid seeds 162 while taking into account expression variation within both species, a model of a single factor with 163 multiple levels (species correspondence: S. peruvianum, S. chilense, and type of seed: normal, hybrid) 164 was implemented in the given RNAseqWrapper module (Schmid 2017). This implies that we contrasted 165 all within-species expression data as one entity (from crosses PP, CC) with all hybrid expression data as 
166 the other entity (from crosses PC, CP). However, to identify possible differences between species, we

167 additionally considered separate within-species comparisons for the DE sRNA clusters (i.e. PP vs. PC,

168 and CC vs. CP). While our main focus is the comparison between intraspecific and failing hybrid seeds,

169 we thus additionally report all DE sRNAs from the two separate contrasts. DE transcripts and sRNAs

170 with more than absolute $2.5 \log$ fold-change and a Bonferroni-corrected $P$ value $<0.05$ are reported as

171 significantly DE.

172 Downstream gene enrichment analyses were carried out using the STRING database (Szklarczyk

173 et al. 2017). We report functional enrichment analyses from STRING with a False Discovery Rate

174 (FDR) of 0.01. When reported, GO assignment was assessed using the PANTHER database (Mi et al.

175 2017). These two databases, STRING and PANTHER, were also used for fine-tuning annotation of

176 genes that lacked annotation in the corresponding SL2.50 ensemble functional annotation files. In the set

177 of DE sRNAs, we defined clusters of genes along the chromosomes based on their curated joint

178 annotation; they were defined as a gene cluster if three or more genes with the same annotation were

179 located within $5 \mathrm{~Kb}$ of genomic space.

181 Expression Mode Classification

182 We compared endosperm expression levels of transcripts and sRNA clusters among S. peruvianum (PP),

183 S. chilense (CC), and their reciprocal hybrids (PC and CP), following the rationale of previous studies to

184 discriminate among the various categories of expression modes (McManus et al. 2010; Combes et al.

185 2015). Independent of whether a gene or sRNA was found to be DE, genes with less than one-fold

186 change between hybrid and normal endosperm were considered as showing conserved expression; for

187 sRNAs, we used a lower threshold of 0.5 -fold expression change. The mode of expression was

188 determined as additive if the expression in the hybrid was less than in S. peruvianum and greater than in

189 S. chilense (or vice versa). If the expression in the hybrid endosperm was similar as in one of the

190 parental species it was classified as dominant for the respective species, and genes and sRNAs with

191 either higher or lower hybrid expression than in both $S$. peruvianum and S. chilense were classified as

192 exhibiting overdominant and underdominant expression, respectively. 


\section{Results}

195 Mapping and Gene Identification

196 We performed sRNA sequencing from whole seeds obtained from intra- and reciprocal inter-specific 197 crosses. Three replicate sets of 'normal' and 'hybrid' sRNA transcriptomes were produced for each of

198 the two main parental plants, the same individuals that were used in our previous study (supplementary

199 figure S1, Supplementary Material online; Florez-Rueda, Paris, et al. 2016). After sequencing, we

200 obtained a mean of 9.6 million reads per library, of which a mean of 7.7 million reads were kept after

201 quality filtering and mapping (supplementary table S1, Supplementary Material online). Not

202 surprisingly, the 24-nt sRNA category was the prevalent type among the 57,711 clusters identified

203 within $2.5 \mathrm{~Kb}$ of annotated genes, whereas 21-22-nt sRNAs and miRNAs accounted for only $10 \%$ of the

204 total identified sRNA clusters (supplementary table S2, Supplementary Material online). To integrate

205 sRNA and gene expression information, we remapped our previously produced endosperm

206 transcriptomes obtained after LAM (Florez-Rueda, Paris, et al. 2016) to the S. lycopersicum reference

207 genome. A mean of 21 million reads per library mapped uniquely to the reference genome and was used

208 in subsequent analyses, making the mean proportion of retained reads $84 \%$ of the initially obtained raw

209 data (supplementary table S1, Supplementary Material online). We thus detected 33,805 transcripts

210 across all endosperm transcriptomes.

Differential Expression in Hybrid Endosperms of Wild Tomatoes

213 We identified common trends of differential expression between normal and hybrid endosperms

214 obtained from both within-species and hybrid crosses, with LA1616A (P) and LA4329B (C) serving as

215 maternal parents in both cross types [contrast (PP, CC) vs. (PC, CP)]. Genes that are consistently DE in

216 the hybrid endosperms of both species tend to have higher levels of expression when compared to

217 'normal' (intraspecific) endosperms in each species (figure 1A). Of the 33,805 transcripts for which we

218 obtained expression values, 2,295 were found as DE in hybrid endosperms; transcripts identified as DE

219 are reported in supplementary table S3, Supplementary Material online. Of these, 1,515 were found

220 overexpressed and 780 underexpressed in hybrid compared to normal endosperms from 

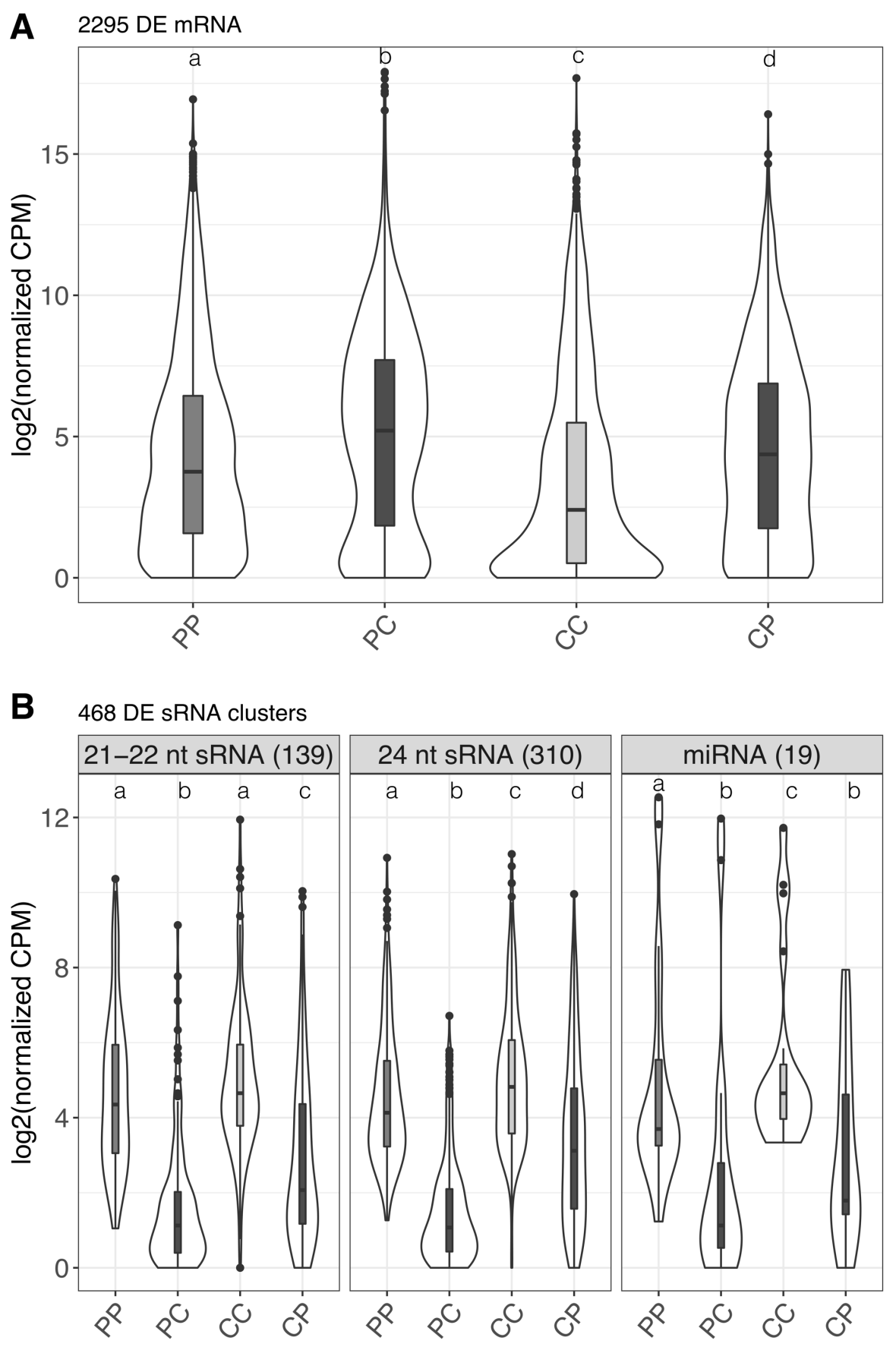

222 FIG. 1.-Expression distributions of 2,295 differentially expressed genes in the endosperm $(A)$, and 468

223 sRNA clusters in whole seeds $(B)$ differentially expressed between within-species and hybrid crosses

224 [contrast (PP, CC) vs. (PC, CP)]. Plants LA1616A (P) and LA4329B (C) served as maternal plants in

225 both cross types. Letters on top of violin plots represent significant differences between expression

226 distributions (Wilcoxon rank-sum test, $P<0.01$ ). 
the same maternal plants.

To test possible roles of sRNAs in mediating HSF and the increases in gene expression (figure $1 A$ )

229 and maternal allelic proportions (Florez-Rueda, Paris, et al. 2016), we investigated patterns of sRNA

230 expression. The pattern of whole-seed sRNA differential expression is in stark contrast to the increase in

231 gene expression we found among DE genes in hybrid endosperms. From the 57,229 total sRNA clusters

232 identified across all sRNA libraries, 468 clusters were DE. These corresponded to miRNAs (19), 24-nt

233 sRNAs (310), and 21-22-nt sRNAs (139) (figure 1B; supplementary table S4, Supplementary Material

234 online). Their altered expression is consistent in reciprocal hybrid crosses, with sRNAs being

235 underexpressed in both PC and CP hybrid seeds. The magnitude of the differences in sRNA expression

236 is larger in seeds from S. peruvianum maternal plants (figure $1 B$ ), thus mirroring the differences in seed

237 phenotype and increases in maternal allelic proportions in hybrid endosperms, which are both more

238 marked in hybrid seeds with S. peruvianum as the seed parent (Florez-Rueda, Paris, et al. 2016; Roth,

239 Florez-Rueda, Griesser, et al. 2018).

240 To shed light on the roles of a putative Pol lV sRNA pathway in Solanum (see Erdmann et al.

241 2017), we examined patterns of expression of the principal subunits of Pol IV, Pol V, and Pol II in the

242 endosperm of hybrid vs normally developing Solanum seeds (supplementary table S5, Supplementary

243 Material online). We observed reduced expression in hybrids of both genes coding for the subunits of

244 Pol lV: RNA polymerase 4 second largest subunit, $R P D 2(\operatorname{logFC}=-0.56$, FDR-corrected $P=0.0148)$

245 and RNA polymerase 4 largest subunit, $R P D 1(\log \mathrm{FC}=-1.82$, FDR-corrected $P=9.69 \mathrm{E}-49)$, as well as

246 reduced expression of the gene coding for subunit $\mathrm{H}$ of RNA polymerase V $(\log \mathrm{FC}=-2.09$, FDR-

$247 \quad$ corrected $P=1.75 \mathrm{E}-55)$.

248 Following the general trend of increased expression in hybrid endosperms (figure 1A), PF00067

249 Cytochrome P450 was enriched with 40 genes found DE (supplementary figure S2A, supplementary

250 table S6, Supplementary Material online). Some of these genes are part of the brassinosteroid

251 biosynthesis KEEG pathway, which is also enriched in the set of genes that show overexpression in

252 hybrid endosperms. Eight genes belonging to the ethylene biosynthetic pathway (GO:0009693)

253 consistently showed increased expression in hybrid endosperms, including $A C O 3, A C O 4, A C O 1$, and

$254 A C S 3$, among other homologs of carboxylate synthases and oxidases in ethylene production

255 (supplementary tables S3 and S6, Supplementary Material online). In addition to genes involved in 
256 ethylene metabolism showing increased expression, 15 ethylene-responsive TF genes were also

257 overexpressed in hybrid endosperms (supplementary figure S2C, Supplementary Material online),

258 contributing to the enrichment of the IPR00147-AP2/ERF domain (supplementary table S6,

259 Supplementary Material online). Genes belonging to this family include highly conserved imprinted

260 genes in Solanum and other plant species (Ikeda 2012; Florez-Rueda, Paris, et al. 2016; Roth, Florez-

261 Rueda, Paris, et al. 2018).

262 The general pattern of overexpression in the hybrid endosperms holds particularly for TF genes

263 (supplementary table S6, supplementary figure S2C-F, Supplementary Material online). Genes

264 encoding subunits of the mediator complex, a global regulator of polymerase II, were found

265 overexpressed in hybrid endosperms, with the term IPR013921 mediator complex significantly enriched.

266 Overexpression was much higher in hybrid endosperm of $S$. chilense than of S. peruvianum maternal

267 plants (supplementary figure S2D, Supplementary Material online), with many of these genes belonging

268 to the term GO:0001104, RNA polymerase II transcription cofactor activity. Strikingly, we uncovered

269 the consistent overexpression of 29 genes containing a MADS-box (IPR002100), likewise displaying

270 more substantial increases of gene expression in hybrid seeds with $S$. chilense maternal plants

271 (supplementary table S6, supplementary figure S2E, Supplementary Material online).

272

273 Joint Signatures of Gene and sRNA Expression Dynamics

274 To investigate the potential role of sRNAs in modulating gene expression in the endosperm, we

275 integrated our seed sRNA data with our endosperm transcriptome data. sRNAs were given the

276 annotation of the gene they mapped to if they fell within $2.5 \mathrm{~kb}$ boundaries (supplementary table S4,

277 Supplementary Material online). Strikingly, the identity of many genes with mapped DE sRNAs

278 revealed roles in epigenetic regulation and/or sRNA biogenesis, suggesting a regulatory feedback

279 mechanism. We identified 119 DE sRNA clusters targeting 79 genes in which underexpression of

280 sRNAs in hybrid seeds was concomitant with the overexpression of the corresponding genes in hybrid

281 endosperms of both cross directions, PC and CP (figure 2). These particular cases suggest gene silencing

282 by the reported clusters of sRNAs that is partly defective in hybrid seeds. Two of these identified genes

283 (Solyc02g091030, Solyc05g012640) encode proteins with RNA and DNA polymerase activity,

284 respectively, and are highly expressed in normal tomato endosperm (figure $2 A, B$ ). The tomato homolog 
285

286

287

288

289

290

291

292

293

294

295

296

297

298

299

300

301

302

303

304

305

306

307

308

309

310

311

312

313

of DEFECTIVE IN MERISTEM SILENCING3 (DMS3), a component of the canonical RdDM pathway in Arabidopsis (Matzke and Mosher 2014), is targeted by DE sRNA clusters in both species (figure 2C), as is a gene encoding a REMORIN protein (Solyc06g035920; figure 2D).

Furthermore, we identified 75 genes targeted by 113 sRNA clusters with asymmetrical changes in gene expression concomitant with reduced sRNA expression in reciprocal PC and CP hybrids (figure 3), highlighting cases of dissimilar epigenetic responses to hybridization. The gene Solyc10g005160, PURINE PERMEASE4 (PUP4) and two clustered LATERAL ORGAN BOUNDARIES (LOB, Solyc09g014700 and Solyc09g014690) genes showed strongly increased expression in the CP hybrid endosperm while their expression in PC hybrid endosperm remained low, concomitant with reduced sRNA expression (figure $3 A, B)$. In contrast, Solyc08g007530, AT-HOOK MOTIF NUCLEARLOCALIZED PROTEIN1 (AHL1), showed reduced gene expression in CP hybrid but increased gene expression in the PC hybrid endosperm (figure 3C), similar to patterns of gene expression of Solyc03g098280 (SlAGO1b), an ARGONAUTE $1 b$ gene (figure 3D). ARGONAUTE proteins are core components of the sRNA-dependent silencing pathways (Matzke and Mosher 2014; Pikaard and Mittelsten Scheid 2014). Six sRNA clusters mapped within the boundaries of this gene; they were less expressed in hybrid seeds, concomitant with higher gene expression in the PC hybrid but slightly decreased gene expression in CP hybrid endosperm (figure 3D).

DE sRNAs mapped to genes arranged in clusters across the tomato genome (figure 4, supplementary tables S4, S7, Supplementary Material online); this led to an increased number of genes per gene class that were consistently targeted by sRNAs. Therefore, the identity of genes that were observed in physical clusters drove our enrichment analyses. These physically linked gene families have undergone expansions in the Solanum lineage compared to Arabidopsis (supplementary tables S6, S7, Supplementary Material online). An example of this pattern are genes belonging to the protein Panther subfamily NUCLEAR TRANSPORT FACTOR 2/RNA RECOGNITION MOTIF PROTEIN (PTHR31413:SF10) with a single member in Arabidopsis (Mi et al. 2017). Thirteen of these genes were targeted mostly by 21-22-nt DE sRNAs, nine of them arranged in clusters on chromosome 2 (supplementary tables S4, S7, Supplementary Material online). Genes belonging to the Panther subfamily PROTEIN FLOWERING LOCUS D (PTHR10742:SF260) have likewise expanded in Solanum, with 14 members in contrast to the single copy in Arabidopsis (Mi et al. 2017). 

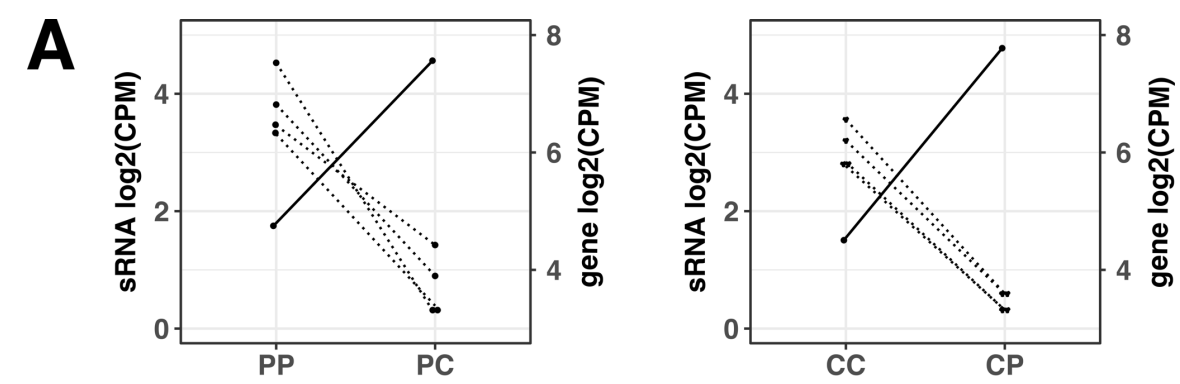

B
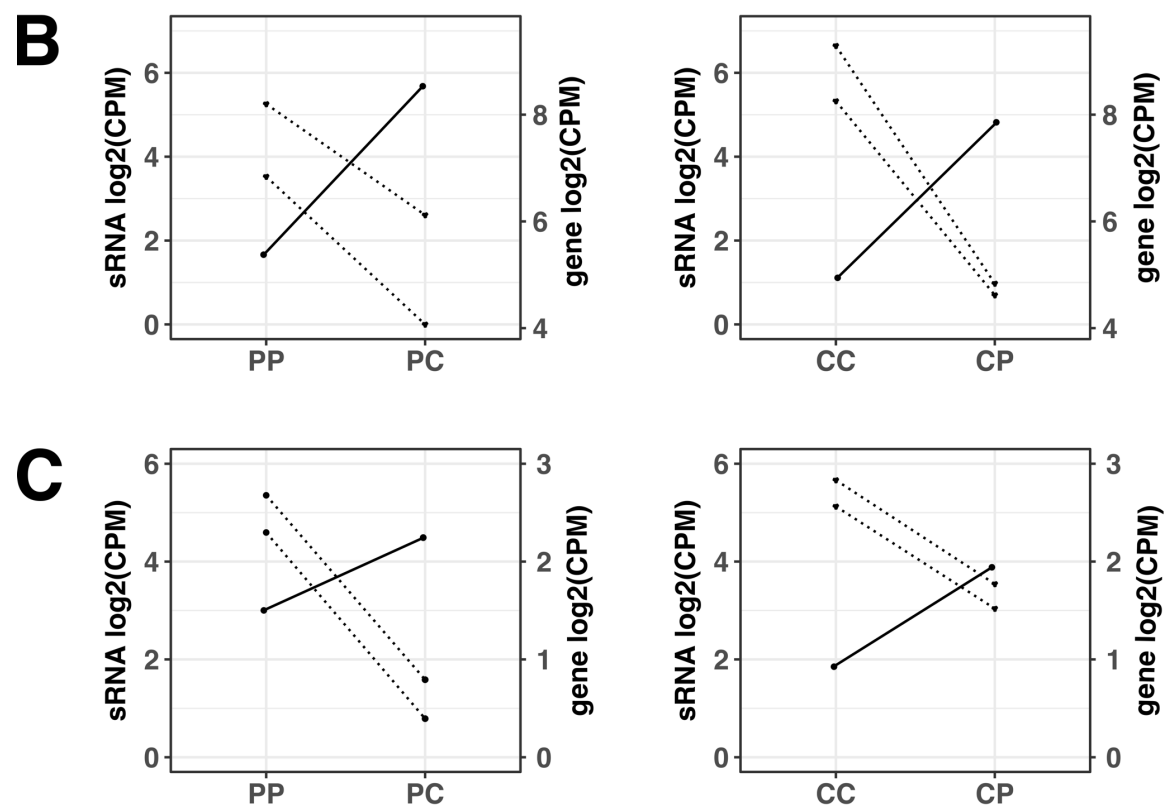

D
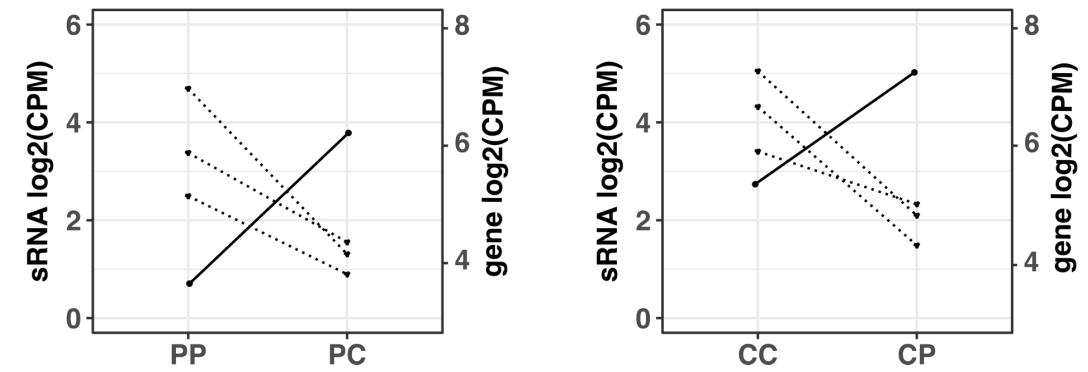

315 FIG. 2.-Examples of decreased sRNA expression being concomitant with gene overexpression in both reciprocal hybrids, indicating that sRNA-mediated gene silencing may be involved. $(A)$ Solyc02g091030, nuclear transcription factor Y subunit C-2 with DNA-directed DNA polymerase activity. (B) Solyc05g012640, T7 RNA polymerase gene 1. (C) Solyc03g083120, DEFECTIVE IN MERISTEM SILENCING3, DMS3. (D) Solyc06g035920, Remorin-1. Dot plots show patterns of expression; on the left $\mathrm{x}$ axis small RNAs expression and on the right $\mathrm{x}$ axis gene expression. Left and right panels show normal and hybrid seeds with $S$. peruvianum $(\mathrm{P})$ and $S$. chilense $(\mathrm{C})$ as maternal plants, respectively. Each dot represents an sRNA cluster and lines within single dot plots trace changes in expression between normally developing and hybrid seeds. Dotted lines and solid lines trace sRNA and gene expression, respectively. All sRNA clusters are significantly DE. Not all genes shown are significantly DE but do have positive fold-changes, indicating gene overexpression in hybrid seeds. 

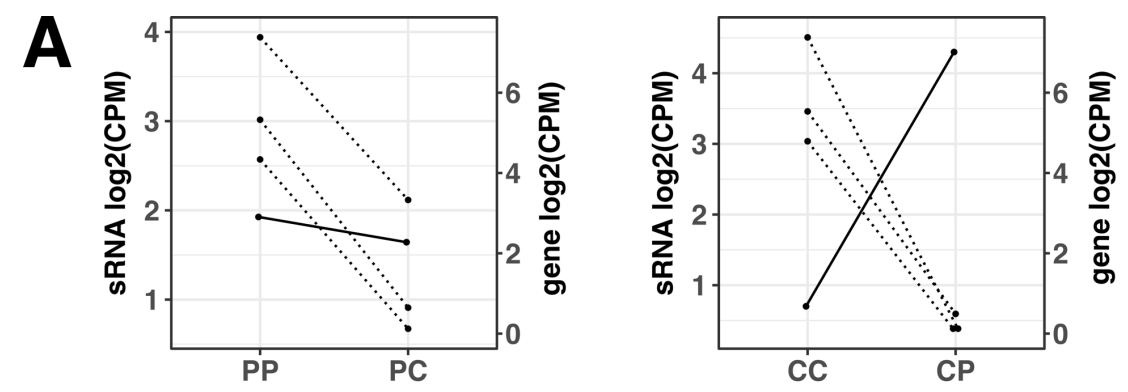

B
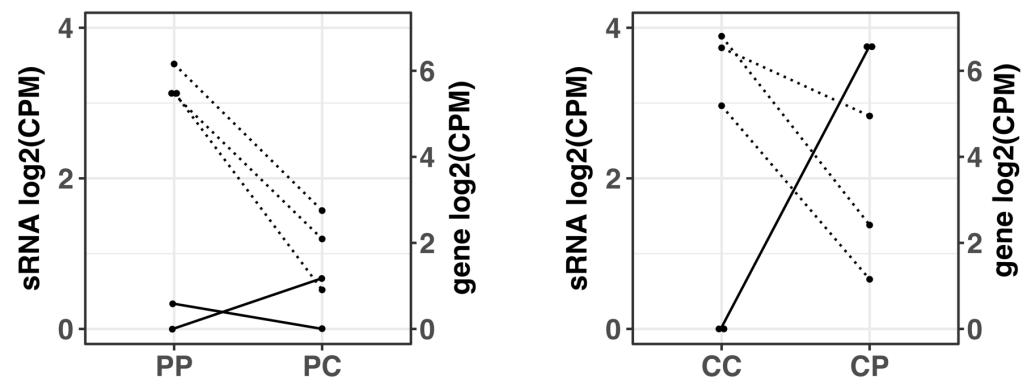

C
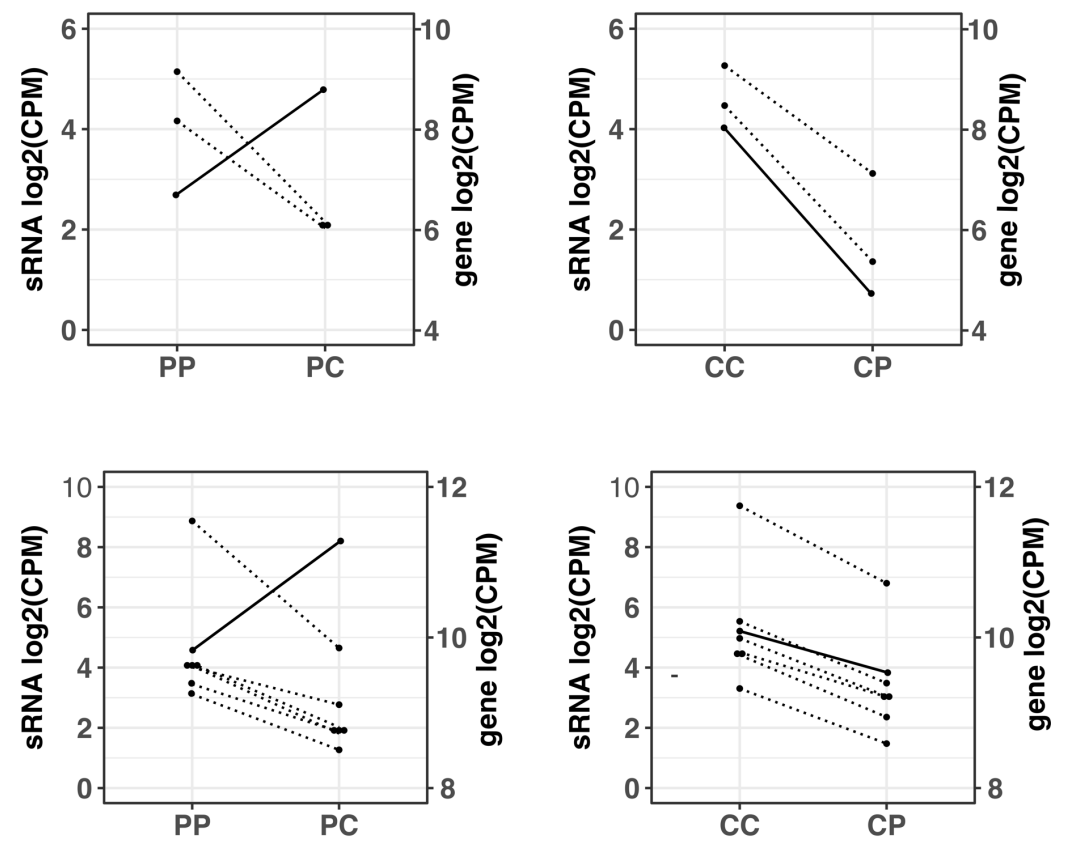

327 FIG. 3.-Examples of sRNA downregulation being concomitant with asymmetrical changes in gene

328 expression in the reciprocal hybrids. (A) Solyc10g005160, PUP4. (B) Solyc09g014700,

329 Solyc09g014690, LOB. (C) Solyc08g007530, AHL1. (D) Solyc03g098280, SlAGO1b, ARGONAUTE

$3301 b$. Panels in each figure part follow the descriptions for Figure 2. 
A

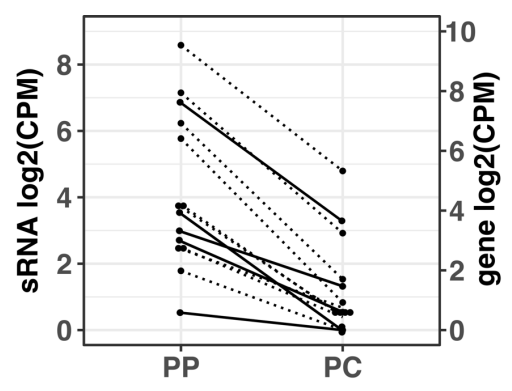

B

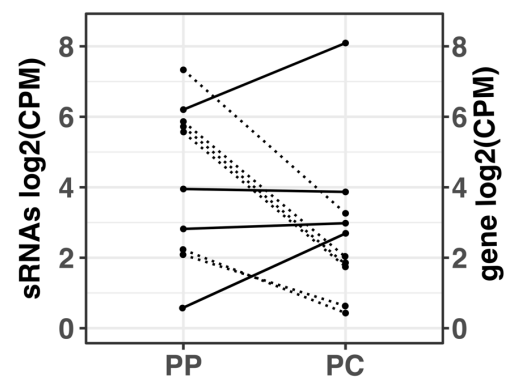

C

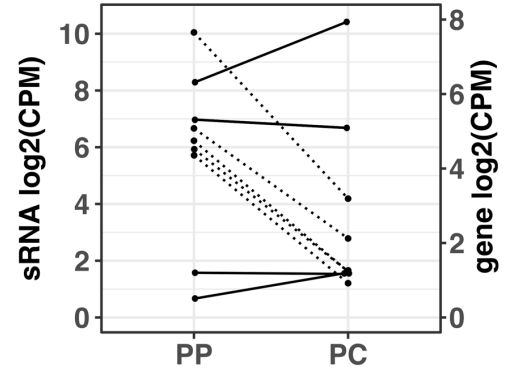

D

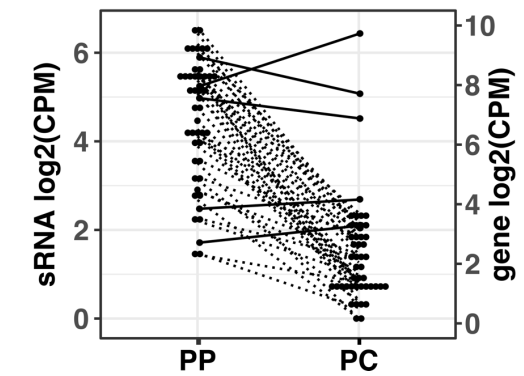

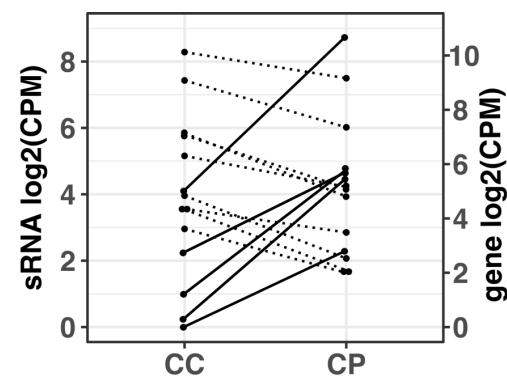
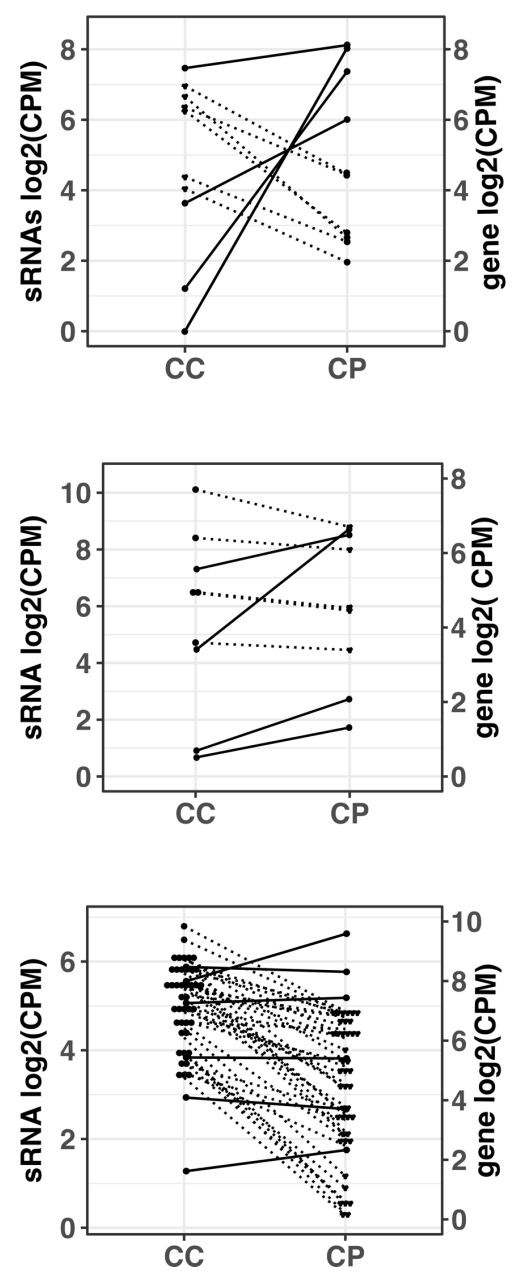

332 FIG. 4.-Examples of DE sRNA target genes arranged in clusters. (A) ARID5 family Solyc12g038750,

333 Solyc12g038740, Solyc12g094730, Solyc12g095740, Solyc01g017030, Solyc01g017790. (B) CLASSY-

3343 family Solyc08g077690, Solyc01g068320, Solyc01g068300, Solyc01g060460. (C) Kinase family

335 protein (D7MB90_ARALY) Solyc12g009370, Solyc12g016120, Solyc12g015990, Solyc05g007140.

336 (D) YIPPEE domain (PTHR13848:SF5) gene family Solyc03g096110, Solyc03g096130,

337 Solyc03g096120, Solyc03g096160, Solyc03g096140, Solyc03g096100, Solyc03g096260,

338 Solyc03g096150, Solyc03g096250, Solyc03g096080. Panels in each figure part follow the descriptions 339 for Figure 2. 
340 We found DE sRNAs mapping to 11 of these genes arranged in clusters on chromosome 5, some of

341 which showed reduced expression levels in hybrid endosperm (supplementary tables S3, S4, S7,

342 Supplementary Material online).

343 Other genes arranged in clusters with sRNAs mapping to them are the chromatin remodelling

344 protein families AT-RICH INTERACTIVE DOMAIN-CONTAINING PROTEIN 5-RELATED

345 (PTHR15348:SF15) (Baba et al. 2011; Chandler et al. 2013), hereafter called ARID5 family, and SNF2

346 DOMAIN-CONTAINING PROTEIN CLASSY 3-RELATED, hereafter called CLASSY3 family

347 (figure $4 A, B$; supplementary table S4, Supplementary Material online). Members of the CLASSY

348 family have putative roles in RdDM (Law et al. 2011) and have recently been shown to be important

349 regulators of sRNA production in Arabidopsis (Zhou et al. 2018). For many of these ARID5 and

350 CLASSY3 family genes, the reduction in sRNA expression in the seed is invariably concurrent with

351 markedly increased gene expression in the CP hybrid endosperm (figure $4 A, B$, right panels). This is not

352 the case in PC hybrid endosperm, in which some genes belonging to the ARID5 family exhibit reduced

353 expression (figure $4 A$ ). Other members of gene families occurring in clusters and targeted by DE sRNAs

354 include members of the Kinase family protein (D7MB90_ARALY) clustered on chromosome 12 (figure

355 4C), and genes with a YIPPEE domain (PTHR13848:SF5) clustered on chromosome 3 (figure 4D). The

356 latter class of genes has been shown to play a role in epigenetic regulation of chromatin, with

357 conditional knockout mouse lines resulting in hypomethylated DNA and embryonic lethality (Kim et al.

358 2012; Subramanian et al. 2016).

359 Some of the genes targeted by DE sRNAs did not exhibit any detectable expression. Lack of

360 expression may indicate that these sRNAs inhibit transcription of these genes, possibly via RdDM or

361 related mechanisms leading to TGS or PTGS (Matzke and Mosher 2014; Pikaard and Mittelsten Scheid

362 2014; Cuerda-Gil and Slotkin 2016). For example, only six of the ten genes encoding a YIPPEE domain

363 targeted by DE sRNAs were expressed in endosperm (figure $4 D$, gene expression). Another example of

364 clustered genes producing DE sRNAs but without detectable gene expression in endosperm is a cluster

365 of DICERLIKE genes located on chromosome 1 (supplementary tables S4, S7, Supplementary Material

366 online). DICER proteins are pivotal components of gene silencing pathways and involved in the

367 cleavage of double-stranded RNA, thus producing siRNAs (Matzke and Mosher 2014; Pikaard and

368 Mittelsten Scheid 2014; Borges and Martienssen 2015; Cuerda-Gil and Slotkin 2016). From the 48 
DICERLIKE genes annotated in the SL2.50 version of the tomato genome, 39 are located in a 5.5-Mb

370

371

372

373

374

375

376

377

378

379

380

381

382

383

384

385

386

387

388

389

390

391

392

393

394

395

396

stretch of chromosome 1, and six of these genes are targeted by significantly DE sRNA clusters in hybrid seeds (supplementary tables S4, S7, Supplementary Material online). Irrespective of the sRNA levels in seeds, there was no detectable endosperm expression of any of these DICER-coding genes clustered on chromosome 1. A single DICER-LIKE 2c gene, Solyc11g008520 located on chromosome 11, was consistently less expressed in hybrid endosperms of both cross directions (supplementary table S3, Supplementary Material online). Another set of genes with mapped DE sRNAs, yet without detectable gene expression (indicating possible sRNA-mediated TGS or PTGS), encodes proteins belonging to the family AMINOTRANSFERASE-LIKE, MOBILE DOMAIN PROTEIN-RELATED (PTHR46033:SF1); 10 genes were found on chromosome 4 with DE sRNA clusters mapping to them (supplementary tables S4, S7, Supplementary Material online). Genes of the same family in Arabidopsis, MAIL1 and MAIN, have recently been shown to be involved in a gene silencing pathway independent of sRNAs and DNA methylation (Ikeda et al. 2017).

\section{Mode of Expression in Hybrids: Dominance May Reflect Differences in Levels of Parental} Conflict

We assessed the mode of expression (conserved, additive, dominant, overdominant, or underdominant) of sRNAs and gene transcripts by comparing total expression levels in S. peruvianum, S. chilense, and their reciprocal hybrids. Following the rationale described in previous studies (McManus et al. 2010; Combes et al. 2015), we performed analyses of expression modes for the DE transcripts and sRNAs as well as for the whole set of transcripts and identified sRNA clusters (figure 5; supplementary figures S3, S4, Supplementary Material online). The analysis of expression modes of all expressed and genes and sRNA clusters (figure $5 A, B$ ) revealed that a large proportion of these show conservation of parental (within-species) expression levels in the hybrids, particularly for gene expression ( $>60 \%$; figure $5 \mathrm{~A}$, purple). While conserved sRNA expression was also the dominant expression mode when evaluating all sRNAs (58.6\% in S. chilense and 56.9\% in S. peruvianum; figure 5B), the entire sRNA data set also revealed a marked pattern of non-conservedness, with maternal dominance being a major category (30.5\% in S. chilense and $28.6 \%$ in S. peruvianum; figure $5 B$ ). 

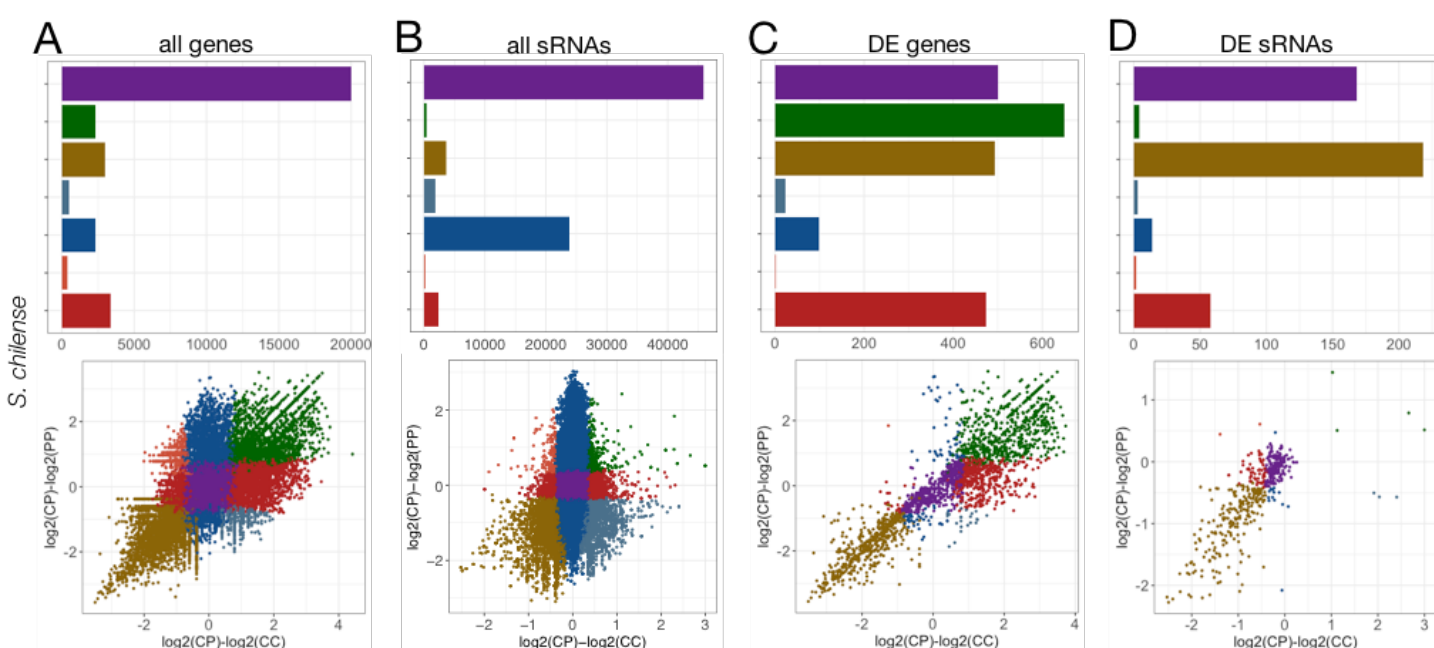

412

398

399

400

401
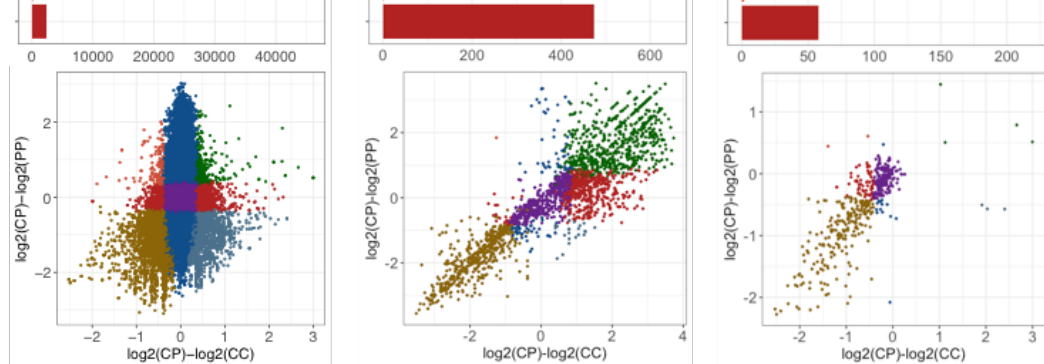

413

402

403
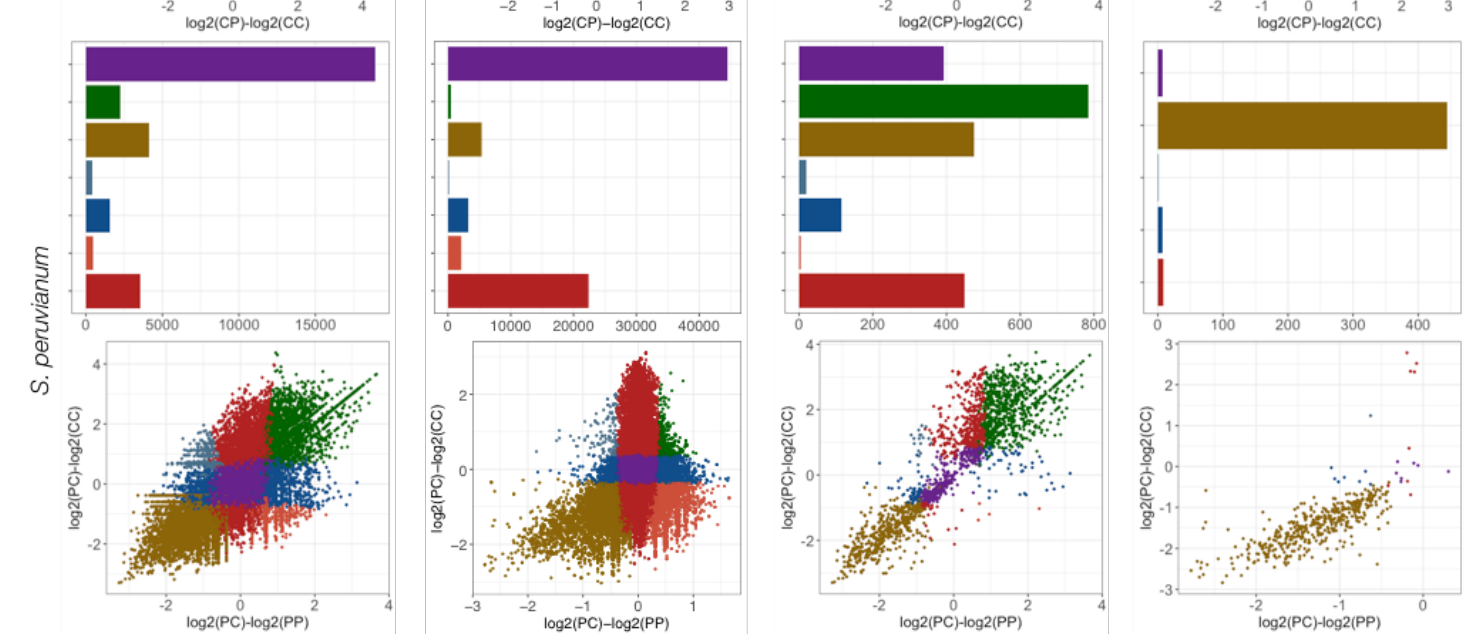

414 FIG. 5.-General patterns of expression

415 modes. Hybrid vs. normal seed

416 comparisons with $S$. chilense (upper

417 panel) and S. peruvianum (lower panel)

418 as maternal plants, respectively. (A) All

419 genes $(n=33,805)$. (B) All sRNA

420 clusters $(n=57,229)$. (C) Differentially

421 expressed genes $(n=2,295)$. (D)

422 Differentially expressed sRNA clusters ( $n$

423 = 468). Expression mode categories are

424 colored as follows: conserved, purple;

425 overdominant, green; underdominant,

426 yellow; $S$. chilense additive, light blue;

427 S.chilense dominant, dark blue; $S$.

428 peruvianum additive, light red; $S$.

429 peruvianum dominant, dark red.

407

408

409

410 
We separately examined the expression modes of 21-22-nt sRNAs, 24-nt sRNAs, and miRNAs for

431 the whole set of sRNAs as well as for DE sRNA clusters (supplementary figures S3, S4, Supplementary

432 Material online). Most of the identified sRNA clusters were 24 nt (supplementary table S2;

433 supplementary figure S3, Supplementary Material online). The levels of non-conserved expression

434 modes were larger in the 21-22-nt category, with maternal dominance reaching $40.5 \%$ in S. peruvianum

435 and $34.7 \%$ in S. chilense. The underdominant class was also larger in the 21-22-nt sRNA category than

436 in the other two categories (supplementary figure S3B, Supplementary Material online).

437 Some DE genes in both species show transgressive expression (figure 5C), with overdominance

438 being the predominant trend followed by underdominance of gene expression. Maternal dominance also

439 markedly contributes to gene expression in the hybrids. An interesting result is the high proportion of

440 genes that are in the $S$. peruvianum-dominant category in CP hybrid seeds, surpassing the maternal-

441 dominant category for $S$. chilense (15.9\% vs. 3.8\%; figure 5C, upper panel). This result suggests that $S$.

442 peruvianum in the paternal role greatly influences gene expression in CP hybrid endosperm despite

443 contributing only one haploid genome. The signature of $S$. peruvianum dominance of gene expression in

444 the CP hybrid is also evident in the expression mode of all genes and not only the DE genes (figure $5 \mathrm{~A}$,

445 upper panel). Although most genes in both cross directions show a conserved pattern of expression, the

446 S. peruvianum dominant category ranks second, surpassing other expression modes $(10.5 \% S$.

447 peruvianum dominance; figure $5 A$, upper panel). These results indicate that the 'genomic dominance' of

448 S. peruvianum relative to $S$. chilense is not restricted to DE genes but acts at a genome-wide level.

449 DE sRNAs were almost completely underdominant in PC hybrid seeds (figure 5D, supplementary

450 figure S4, Supplementary Material online). In contrast, many DE sRNA clusters in CP hybrid seeds

451 showed conserved expression. The S. peruvianum-dominant signature evident in the mode of gene

452 expression (figure $5 \mathrm{~A}, \mathrm{C}$ ) is also apparent in the expression mode of DE sRNA clusters in CP hybrid

453 seeds, with $11.6 \%$ of the DE sRNA clusters falling into this category. However, the most striking trend

454 in the expression mode of sRNAs was that of underdominance of DE sRNA clusters in hybrid seeds

455 (figure 5D, supplementary figure S4, Supplementary Material online). 


\section{Discussion}

458 Evidence for Conserved Epigenetic Landscapes in Compromised Hybrid Endosperm

459 Our analyses of sRNAs and transcripts that were differentially expressed between normal and failing

460 seeds/endosperms revealed striking similarities with previous work on transcriptomic responses to

461 hybridization in other taxa, particularly with the effects of Pol IV mutations on the epigenomic

462 landscape of Arabidopsis endosperm. Erdmann et al. (2017) demonstrated that the Pol IV sRNA

463 pathway mediates dosage interactions between maternal and paternal genomes. Specifically, they

464 showed that disabling mutations in $n r p d 1$ led to shifts toward higher expression of maternally inherited

465 alleles. These results mirror our previous findings of increased maternal expression proportions in

466 Solanum hybrid endosperms (Florez-Rueda, Paris, et al. 2016). Likewise, Erdmann et al. (2017) reported

467 increased gene expression in $n r p d l$ mutant endosperm compared to WT, resembling the increased gene

468 expression among DE genes in Solanum hybrid endosperms (figure $1 A$ ).

469 Taken together, the reduction in Pol IV expression and the overall increase in expression of DE

470 transcripts and maternal expression proportions in hybrids (Florez-Rueda, Paris, et al. 2016) allows us to

471 draw comparisons between effects of the Arabidopsis nrpdl mutant (Erdmann et al. 2017) and the

472 natural case of HSF we explore here in Solanum. Based on these obvious parallels, we postulate a

473 Solanum Pol IV sRNA pathway acting in a similar fashion to that described in Arabidopsis (Erdmann et

474 al. 2017), mediating dosage interactions of the parental genomes upon fertilization. We propose that the

475 Pol IV sRNA pathway serves to maintain the expected 2:1 ratio in the endosperm, likely through direct

476 and/or indirect effects on many genes in the endosperm. The observed reduced expression of the main

477 Pol IV subunits may be functionally linked to the increased maternal expression proportions in hybrid

478 endosperm of wild tomatoes (Florez-Rueda, Paris, et al. 2016).

Increased expression of MADS-box TF genes upon hybridization has previously been reported in

480 Arabidopsis (Josefsson et al. 2006; Walia et al. 2009; Hehenberger et al. 2012; Lu et al. 2012; Burkart-

481 Waco et al. 2013), Capsella (Rebernig et al. 2015), and Oryza (Ishikawa et al. 2011). We found a large

482 number of MADS-box genes (among other TF genes) overexpressed in both reciprocal hybrid

483 endosperms (supplementary figure S2E, supplementary table S3, Supplementary Material online).

484 MADS-domain TFs have been shown to play key regulatory roles in plant reproduction, in particular in

485 regulating female gametophyte, embryo, and endosperm development (reviewed in Masiero et al. 2011). 
Likewise, AGAMOUS-LIKE (AGL) genes were jointly overexpressed in 'paternal-excess-like' crosses

488 protein dimerization activity (GO:0046983) and include 11 AGL genes, 13 2FE-2S FERREDOXIN-

489 LIKE genes, three PHERES genes, APETALLA3 and SEPALATA3, among others (supplementary tables

490 S3, S6, Supplementary Material online). AGL proteins, which themselves are MADS-domain proteins,

491 have been shown to affect endosperm development in Arabidopsis (Kang et al. 2008; Shirzadi et al.

492 2011). Intriguingly, overexpression of $A G L 62$ and $A G L 90$ is associated with the postzygotic barrier

493 between $A$. thaliana and A. arenosa, which manifests as endosperm over-proliferation and delayed

494 development (Josefsson et al. 2006; Walia et al. 2009; Burkart-Waco et al. 2013). Providing functional

495 validation for this pattern, transgenic underexpression of AGL62 attenuated the level of HSF in

496 Arabidopsis (Hehenberger et al. 2012).

497 sRNAs have been shown to modulate expression of MADS-box TF genes; maternal small

498 interfering RNA (siRNA) expression is negatively correlated with $A G L$ gene expression in Arabidopsis

499 endosperm (Lu et al. 2012). However, our analyses did not support a consistent trend of sRNAs

500 targeting MADS-box TF genes; we found but a single gene Solyc12g056460 (SOC1) with an associated

501 DE sRNA cluster (supplementary table S4, Supplementary Material online). Taken together, this and

502 earlier Arabidopsis studies suggest that the putative functions of MADS-domain TFs in mediating both

503 normal seed development and endosperm-based HSF are conserved across angiosperms. Specific

504 functions of MADS-box TF genes in Solanum have not yet been studied, but here we have uncovered a

505 list of candidates with potentially important roles that remain to be functionally validated.

Qualitative and quantitative sRNA differences between the parental genomes may affect the

507 expression of genes and TEs neighboring the sRNAs in hybrid seeds. In some instances of hybridization,

508 changes in sRNA expression are concomitant with heterosis (Groszmann et al. 2011; Barber et al. 2012),

509 although a causal role of sRNAs has not been shown; in our Solanum case and others, they may lead to

510 HSF (Ng et al. 2012; Kirkbride et al. 2015; Florez-Rueda, Paris, et al. 2016; Garner et al. 2016). We

511 found that DE sRNAs were consistently underexpressed in hybrid seeds (figure $1 B$ ); this trend is

512 reflected in underdominance of sRNA expression in hybrid seeds when compared to seeds derived from

513 intraspecific crosses on the same maternal plant. Underdominance of sRNA expression upon

514 hybridization has been reported in other tissues besides the seed and in hybridization cases in diverse 
515 plant genera (Groszmann et al. 2011; Barber et al. 2012; Lu et al. 2012; Shen et al. 2012; Shivaprasad et

516 al. 2012; He et al. 2013). In all these cases, as well as ours, the molecular mechanisms leading to

517 reduced sRNA levels are still unknown; based on the reduced expression of Pol IV subunits

518 (supplementary table S4, Supplementary Material online), we can hypothesize that perturbations in the

519 Pol lV sRNA pathway may be involved (Erdmann et al. 2017).

520 We uncovered high levels of maternal dominance of sRNA expression that may be explained by

521 the nature of the seed tissue we collected (seeds extracted manually with subsequent washes), maternal

522 seed coat being one of its components. Another possible scenario is that the sRNAs exhibiting maternal

523 dominance may be generated by filial seed tissues. However, there is disagreement among studies in $A$.

524 thaliana whether 24-nt siRNAs show strongly maternally-biased expression (Mosher et al. 2009;

525 Erdmann et al. 2017; Kirkbride et al. 2019). Regardless, these sRNAs are thought to accumulate in the

526 endosperm and to mediate gene expression (Calarco and Martienssen 2011); the high level of observed

527 maternal dominance in the expression inheritance of sRNAs in both species suggests that this may also

528 be the case in Solanum.

529

530 Feedback Regulation of Core Silencing Proteins through sRNA-mediated Silencing

531 Our data suggest that sRNAs that are DE in hybrid seeds target many genes with important functions in 532 sRNA biogenesis and epigenetic regulation. Importantly, members of the ARID5 and CLASSY3 families,

533 DICER, $A G O 1 B, A G O 5, R D M 1$, and $D M S 3$ were shown to be associated with sRNAs in tomato seeds in

534 abundances that were significantly different in both PC and CP hybrid seeds (figures 2-4,

535 supplementary table S4, Supplementary Material online). For some of these genes, we were able to

536 additionally assess gene expression levels; the apparent effect of sRNA abundance on gene expression

537 suggests that sRNA-mediated gene silencing impacts the expression of some of these genes and may be

538 defective in hybrid seeds, plausibly contributing to HSF. We hypothesize that these genes, some of

539 which are regulators of TGS or PTGS themselves, are subject to feedback regulation orchestrated by

540 their own sRNA products. Negative feedback regulation of DICERLIKE genes has been described in

541 Arabidopsis (Xie et al. 2003; Bologna and Voinnet 2014) and yeast (Oberti et al. 2015); such feedback

542 regulation is thought to allow homeostatic control of the cellular silencing machinery (Bologna and

543 Voinnet 2014). The only gene for which we detected an effect on allele-specific expression is the 
544 ARGONAUTE-encoding gene Solyc03g098280, SlAGO1b (figure 3D). As a paternally expressed gene

545 (PEG) with a low maternal proportions in normal endosperm of S. peruvianum, this gene showed the

546 'typical' increase (from 0.25 to 0.87 maternal proportion) in PC hybrid endosperm that we previously

547 uncovered for the majority of PEGs (Florez-Rueda, Paris, et al. 2016). We posit that the observed

548 underexpression of sRNA clusters mapping to $S l A G O 1 b$ and its flanking regions may be responsible for

549 its increased gene expression, with a higher maternal proportion in hybrid endosperm derived from

550 maternal S. peruvianum.

551 Although we cannot provide functional verification to support feedback regulation of genes

552 involved in sRNA-mediated gene silencing in Solanum endosperm, our results provide pioneering

553 glimpses into the epigenetic landscape in the context of HSF. We could show that DE sRNA clusters

554 map to genes playing pivotal roles in epigenetic regulation, with expected implications for HSF. Further

555 characterization of the epigenomic landscape of the endosperm through chromatin immunoprecipitation

556 and sequencing (ChIP-seq) as well as methylome sequencing will allow a proper evaluation of these

557 hypotheses.

558

Mode of Expression in Hybrids: Dominance may reflect Differences in Parental Conflict

560 Previous analyses of expression modes have been restricted to evaluating inheritance in whole plants

561 that were successful hybridization products of within- or among-species crosses (Eichten et al. 2011; Shi

562 et al. 2012; Bell et al. 2013; Combes et al. 2015; Li et al. 2015; Carlson et al. 2017). Although these

563 types of analyses on whole hybrid plants provide valuable insights into the transcriptomic effects of

564 hybridization, they do not address the issue of parental conflict that is expected to play out in the

565 developing seed (Haig and Westoby 1991; Haig 2013; Lafon-Placette and Köhler 2016).

566 The near-complete HSF phenotype characterizing both cross directions between S. peruvianum

567 and $S$. chilense (yet with marked phenotypic differences between reciprocal crosses) may be seen as

568 resulting from different levels of parental conflict among diverged parental lineages (Haig and Westoby

569 1989; Brandvain and Haig 2005; Haig 2013). Hybrid seeds from S. chilense maternal plants (CP) are

570 larger, showing a 'paternal excess-like' phenotype in contrast to the smaller hybrid seeds with $S$.

571 peruvianum mothers (PC) that show a 'maternal excess-like' phenotype (Florez-Rueda 2014; Florez-

572 Rueda, Paris, et al. 2016; Roth, Florez-Rueda, Griesser, et al. 2018). The reciprocal differences both in 
573 early seed development and mature hybrid seed size suggest that the S. peruvianum lineage has evolved

574 under higher levels of parental conflict than has S. chilense. These patterns and inferences are consistent

575 with higher range-wide nucleotide diversity, indicative of higher effective population size (Städler et al.

576 2008; Tellier et al. 2011; Beddows et al. 2017), and higher expression levels of imprinted genes in $S$.

577 peruvianum (Roth, Florez-Rueda, Paris, et al. 2018).

578 Likewise, S. peruvianum drives expression landscape polarization in hybrid endosperms derived

579 from reciprocal crosses with $S$. chilense and $S$. arcanum (Roth et al. 2019). In line with these

580 observations, our analyses of the expression modes of DE sRNAs and genes revealed a trend of $S$.

581 peruvianum dominance in $\mathrm{CP}$ hybrid seeds and endosperm, respectively (figure $5 C, D$, upper panel).

582 This signature held true not only for DE genes and sRNAs but was also detected at a genome wide-level,

583 specifically on the larger dataset of all expressed genes where the S. peruvianum-dominant category

584 ranked second (figure 5A, upper panel). We interpret the pattern of $S$. peruvianum dominance as

585 consistent with the rationale of the weak inbreeder/strong outbreeder (WISO) hypothesis (Brandvain and

586 Haig 2005), with the S. peruvianum genome 'overpowering' that of S. chilense, which putatively

587 evolved under lower levels of parental conflict. These inferences are in accordance with our prior and

588 current evidence for higher effective ploidy of S. peruvianum compared to $S$. chilense (Roth et al. 2019),

589 and how it plausibly underpins seeds' developmental and phenotypic differences between these two wild

590 tomato lineages: smaller hybrid seed size and transcriptomic signals (Florez-Rueda, Paris, et al. 2016;

591 Roth, Florez-Rueda, Griesser, et al. 2018; Roth et al. 2019), such as a higher proportion of DE genes,

592 DE sRNAs, and larger shifts toward maternal allelic expression in PC (compared to CP) hybrid

593 endosperm.

594

595

Data Availability

596 Raw sequence data for the RNA-sequencing dataset used in this study are available from the Sequence

597 Read Archive (https://race.ncbi.nlm.nih.gov/Traces/sra/) with the accession numbers XXXXXXXXX

598 (sRNAs; this study) and SRX1850236 (mRNA; Florez-Rueda, Paris, et al. 2016).

599

600 


\section{Acknowledgments}

602 We are grateful to Maja Frei and Esther Zürcher for taking expert care of the plants, to Margot Paris and

603 Anja Schmidt for technical advice, and to Alex Widmer for general support of this project. We thank the

604 C.M. Rick Tomato Genetics Resource Center at U.C. Davis for generously supplying seed samples, and

605 Lennart Opitz for bioinformatics support. We also thank the Genetic Diversity Center (ETH Zurich,

606 Switzerland) and the Swiss Institute for Bioinformatics (Lausanne, Switzerland) for providing valuable

607 tools and training for bioinformatics analyses. This work was supported by the University of Zurich, the

608 ETH Zurich, and grants from the Swiss National Science Foundation [grant number 31003A_130702 to

609 T.S., 310030B_160336 to U.G.], an ETH Research Grant [grant number ETH-40 13-2 to T.S. and Alex

610 Widmer], and the University of Zurich Research Priority Program Evolution in Action [Pilot Grant to

611 A.M.F.-R. and U.G.].

612

613 Literature Cited

614 Axtell MJ. 2013. ShortStack: comprehensive annotation and quantification of small RNA genes. RNA $615 \quad 19: 740-751$.

616 Baba A, et al. 2011. PKA-dependent regulation of the histone lysine demethylase complex PHF2-

617 ARID5B. Nat Cell Biol. 13:668-675.

618 Barber WT, et al. 2012. Repeat associated small RNAs vary among parents and following hybridization 619 in maize. Proc Natl Acad Sci U S A. 109:10444-10449.

620 Beamish K. 1955. Seed failure following hybridization between the hexaploid Solanum demissum and 621 four diploid Solanum species. Am J Bot. 42:297-304.

622 Beddows I, Reddy A, Kloesges T, Rose LE. 2017. Population genomics in wild tomatoes - the interplay 623 of divergence and admixture. Genome Biol Evol. 9:3023-3038.

Bell GDM, Kane NC, Rieseberg LH, Adams KL. 2013. RNA-Seq analysis of allele-specific expression, 625 hybrid effects, and regulatory divergence in hybrids compared with their parents from natural 626 populations. Genome Biol Evol. 5:1309-1323.

627 Benkovics AH, Timmermans MCP. 2014. Developmental patterning by gradients of mobile small 628 RNAs. Curr Opin Genet Dev. 27:83-91.

629 Berger F. 2003. Endosperm: the crossroad of seed development. Curr Opin Plant Biol. 6:42-50.

630 Bologna NG, Voinnet O. 2014. The diversity, biogenesis, and activities of endogenous silencing small 
RNAs in Arabidopsis. Annu Rev Plant Biol. 65:473-503.

632 Borges F, Martienssen RA. 2015. The expanding world of small RNAs in plants. Nat Rev Mol Cell 633 Biol. 16:727-741.

634 Borges $\mathrm{F}$, et al. 2018. Transposon-derived small RNAs triggered by miR845 mediate genome dosage response in Arabidopsis. Nat Genet. 50:186-192.

636 Bourc'his D, Voinnet O. 2010. A small-RNA perspective on gametogenesis, fertilization, and early 637 zygotic development. Science 330:617-622.

638 Brandvain Y, Haig D. 2005. Divergent mating systems and parental conflict as a barrier to hybridization 639 in flowering plants. Am Nat. 166:330-338.

640 Briggs CL. 1993. Endosperm development in Solanum nigrum L. Formation of the zone of separation 641 and secretion. Ann Bot. 72:303-313.

642 Burkart-Waco D, Ngo K, Dilkes B, Josefsson C, Comai L. 2013. Early disruption of maternal-zygotic 643 interaction and activation of defense-like responses in Arabidopsis interspecific crosses. Plant Cell 25:2037-2055.

645 Calarco JP, Martienssen RA. 2011. Genome reprogramming and small interfering RNA in the 646 Arabidopsis germline. Curr Opin Genet Dev. 21:134-139.

647 Carlson $\mathrm{CH}$, et al. 2017. Dominance and sexual dimorphism pervade the Salix purpurea L.

$648 \quad$ transcriptome. Genome Biol Evol. 9:2377-2394.

649 Chandler RL, et al. 2013. ARID1a-DNA interactions are required for promoter occupancy by SWI/SNF. $650 \quad$ Mol Cell Biol. 33:265-280.

651 Combes MC, et al. 2015. Regulatory divergence between parental alleles determines gene expression patterns in hybrids. Genome Biol Evol. 7:1110-1121.

653 Cooper DC, Brink RA. 1945. Seed collapse following matings between diploid and tetraploid races of 654 Lycopersicon pimpinellifolium. Genetics 30:376-401.

655 Coyne JA, Orr HA. 2004. Speciation. Sunderland, MA: Sinauer Associates.

656 Cuerda-Gil D, Slotkin RK. 2016. Non-canonical RNA-directed DNA methylation. Nat Plants 2:16163.

657 D’Ario M, Griffiths-Jones S, Kim M. 2017. Small RNAs: big impact on plant development. Trends $658 \quad$ Plant Sci. 22:1056-1068.

659 Dilkes BP, et al. 2008. The maternally expressed WRKY transcription factor TTG2 controls lethality in 660 interploidy crosses of Arabidopsis. PLoS Biol. 6:2707-2720.

661 Dobin A, et al. 2013. STAR: ultrafast universal RNA-seq aligner. Bioinformatics 29:15-21. 
662 Eichten SR, et al. 2011. Heritable epigenetic variation among maize inbreds. PLoS Genet. 7:e1002372.

663 Erdmann RM, Satyaki PRV, Klosinska M, Gehring M. 2017. A small RNA pathway mediates allelic 664 dosage in endosperm. Cell Rep. 21:3364-3372.

665 Florez-Rueda AM. 2014. Postzygotic barriers to interbreeding in wild tomatoes: genomic imprinting and 666 transcriptional signatures of hybrid seed failure. PhD dissertation, ETH Zurich, Switzerland.

667 Florez-Rueda AM, Paris M, et al. 2016. Genomic imprinting in the endosperm is systematically 668 perturbed in abortive hybrid tomato seeds. Mol Biol Evol. 33:2935-2946.

669 Florez-Rueda AM, Grossniklaus U, Schmidt A. 2016. Laser-assisted microdissection (LAM) as a tool 670 for transcriptional profiling of individual cell types. J Vis Exp. 111:e53916.

671 Fultz D, Choudury SG, Slotkin RK. 2015. Silencing of active transposable elements in plants. Curr Opin 672 Plant Biol. 27:67-76.

673 Garner AG, Kenney AM, Fishman L, Sweigart AL. 2016. Genetic loci with parent-of-origin effects 674 cause hybrid seed lethality in crosses between Mimulus species. New Phytol. 211:319-331.

Groszmann M, et al. 2011. Changes in 24-nt siRNA levels in Arabidopsis hybrids suggest an epigenetic contribution to hybrid vigor. Proc Natl Acad Sci U S A. 108:2617-2622.

Gutierrez-Marcos JF, Pennington PD, Costa LM, Dickinson HG. 2003. Imprinting in the endosperm: a possible role in preventing wide hybridization. Phil Trans R Soc Biol Sci. 358:1105-1111.

Haig D. 2013. Kin conflict in seed development: an interdependent but fractious collective. Annu Rev Cell Dev Biol. 29:189-211.

Haig D, Westoby M. 1989. Parent-specific gene expression and the triploid endosperm. Am Nat. 134:147-155.

Haig D, Westoby M. 1991. Genomic imprinting in endosperm: its effect on seed development in crosses between species, and between different ploidies of the same species, and its implications for the evolution of apomixis. Phil Trans R Soc Biol Sci. 333:1-13.

$\mathrm{He} \mathrm{G}$, et al. 2013. Conservation and divergence of transcriptomic and epigenomic variation in maize hybrids. Genome Biol. 14:R57.

Hehenberger E, Kradolfer D, Köhler C. 2012. Endosperm cellularization defines an important developmental transition for embryo development. Development 139:2031-2039. mechanisms. Plant Cell Physiol. 53:809-816.

Ikeda Y, et al. 2017. Arabidopsis proteins with a transposon-related domain act in gene silencing. Nat 
Ishikawa R, et al. 2011. Rice interspecies hybrids show precocious or delayed developmental transitions in the endosperm without change to the rate of syncytial nuclear division. Plant J. 65:798-806. endosperm development in interspecific crosses. Theor Appl Genet. 57:5-9.

Josefsson C, Dilkes B, Comai L. 2006. Parent-dependent loss of gene silencing during interspecies hybridization. Curr Biol. 16:1322-1328.

Jullien P, Berger F. 2010. Parental genome dosage imbalance deregulates imprinting in Arabidopsis. PLoS Genet. 6:e1000885.

Kang I-H, Steffen JG, Portereiko MF, Lloyd A, Drews GN. 2008. The AGL62 MADS domain protein regulates cellularization during endosperm development in Arabidopsis. Plant Cell 20:635-647.

Kim IS, et al. 2012. Roles of Mis18 $\alpha$ in epigenetic regulation of centromeric chromatin and CENP-A loading. Mol Cell. 46:260-273.

Kirkbride RC, et al. 2015. An epigenetic role for disrupted paternal gene expression in postzygotic seed abortion in Arabidopsis interspecific hybrids. Mol Plant 8:1766-1775.

Kirkbride RC, et al. 2019. Maternal small RNAs mediate spatial-temporal regulation of gene expression, imprinting, and seed development in Arabidopsis. Proc Natl Acad Sci U S A. 116:2761-2766.

Lafon-Placette C, Köhler C. 2016. Endosperm-based postzygotic hybridization barriers: developmental mechanisms and evolutionary drivers. Mol Ecol. 25:2620-2629.

713 in Capsella. Nat Plants 4:352-357.

714 Law JA, Vashisht AA, Wohlschlegel JA, Jacobsen SE. 2011. SHH1, a homeodomain protein required 715 for DNA methylation, as well as RDR2, RDM4, and chromatin remodeling factors, associate with 716 RNA Polymerase IV. PLoS Genet. 7:e1002195.

717 Li Q, Li Y, Moose SP, Hudson ME. 2015. Transposable elements, mRNA expression level and strand718 specificity of small RNAs are associated with non-additive inheritance of gene expression in hybrid plants. BMC Plant Biol. 15:168.

721 data with DESeq2. Genome Biol. 15:550.

722 Lu J, Zhang C, Baulcombe DC, Chen ZJ. 2012. Maternal siRNAs as regulators of parental genome 723 imbalance and gene expression in endosperm of Arabidopsis seeds. Proc Natl Acad Sci U S A. 
109:5529-5534.

Martínez G, Köhler C. 2017. Role of small RNAs in epigenetic reprogramming during plant sexual reproduction. Curr Opin Plant Biol. 36:22-28.

Martínez G, Panda K, Köhler C, Slotkin RK. 2016. Silencing in sperm cells is directed by RNA movement from the surrounding nurse cell. Nat Plants 2:16030.

Martínez G, et al. 2018. Paternal easiRNAs regulate parental genome dosage in Arabidopsis. Nat Genet. 50:193-198.

Masiero S, Colombo L, Grini PE, Schnittger A, Kater MM. 2011. The emerging importance of type I MADS box transcription factors for plant reproduction. Plant Cell 23:865-872.

Matzke MA, Mosher RA. 2014. RNA-directed DNA methylation: an epigenetic pathway of increasing complexity. Nat Rev Genet. 15:394-408.

McManus CJ, et al. 2010. Regulatory divergence in Drosophila revealed by mRNA-seq. Genome Res. 20:816-825.

Mi H, et al. 2017. PANTHER version 11: expanded annotation data from gene ontology and reactome pathways, and data analysis tool enhancements. Nucleic Acids Res. 45:D183-D189.

Mosher RA, et al. 2009. Uniparental expression of PolIV-dependent siRNAs in developing endosperm of Arabidopsis. Nature 460:283-286.

Ng DWK, Lu J, Chen ZJ. 2012. Big roles for small RNAs in polyploidy, hybrid vigor, and hybrid incompatibility. Curr Opin Plant Biol. 15:154-161.

Oberti D, et al. 2015. Dicer and Hsp104 function in a negative feedback loop to confer robustness to environmental stress. Cell Rep. 10:47-61.

Oneal E, Willis JH, Franks RG. 2016. Disruption of endosperm development is a major cause of hybrid seed inviability between Mimulus guttatus and M. nudatus. New Phytol. 210:1107-1120.

Ortiz R, Ehlenfeldt MK. 1992. The importance of endosperm balance number in potato breeding and the evolution of tuber-bearing Solanum species. Euphytica 60:105-113.

Pikaard CS, Mittelsten Scheid O. 2014. Epigenetic regulation in plants. Cold Spring Harb Perspect Biol. 6:a019315.

752 Bioinformatics 26:841-842.

753 R Development Core Team. 2014. R: a language and environment for statistical computing. R Found 754 Stat Comput Vienna, Austria. URL http://www.R-project.org/. 
Rebernig CA, Lafon-Placette C, Hatorangan MR, Slotte T, Köhler C. 2015. Non-reciprocal interspecies hybridization barriers in the Capsella genus are established in the endosperm. PLoS Genet. 11:e1005295.

Rick CM. 1963. Barriers to interbreeding in Lycopersicon peruvianum. Evolution 17:216-232.

Rick CM, Lamm R. 1955. Biosystematic studies on the status of Lycopersicon chilense. Am J Bot. $42: 663-675$.

Rodrigues JA, et al. 2013. Imprinted expression of genes and small RNA is associated with localized hypomethylation of the maternal genome in rice endosperm. Proc Natl Acad Sci U S A. 110:79347939.

Roth M, Florez-Rueda AM, Griesser S, Paris M, Städler T. 2018. Incidence and developmental timing of endosperm failure in post-zygotic isolation between wild tomato lineages. Ann Bot. 121:107118.

Roth M, Florez-Rueda AM, Paris M, Städler T. 2018. Wild tomato endosperm transcriptomes reveal common roles of genomic imprinting in both nuclear and cellular endosperm. Plant J. 95:10841101.

Roth M, Florez-Rueda AM, Städler T. 2019. Differences in effective ploidy drive genome-wide endosperm expression polarization and seed failure in wild tomato hybrids. Genetics 212:141-152.

Schmid MW. 2017. RNA-Seq data analysis protocol: combining in-house and publicly available data. In: Schmidt A, editor. Plant Germline Development: Methods and Protocols. New York: Springer. p. 309-335.

Scott RJ, Spielman M, Bailey J, Dickinson HG. 1998. Parent-of-origin effects on seed development in Arabidopsis thaliana. Development 125:3329-3341.

Shen H, et al. 2012. Genome-wide analysis of DNA methylation and gene expression changes in two Arabidopsis ecotypes and their reciprocal hybrids. Plant Cell 24:875-892.

Shi X, et al. 2012. Cis- and trans-regulatory divergence between progenitor species determines geneexpression novelty in Arabidopsis allopolyploids. Nat Commun. 3:229-237.

Shirzadi R, et al. 2011. Genome-wide transcript profiling of endosperm without paternal contribution identifies parent-of-origin-dependent regulation of AGAMOUS-LIKE36. PLoS Genet. 7:e1001303.

Shivaprasad PV, Dunn RM, Santos BA, Bassett A, Baulcombe DC. 2012. Extraordinary transgressive phenotypes of hybrid tomato are influenced by epigenetics and small silencing RNAs. EMBO J. $31: 257-266$. 
786 Städler T, Arunyawat U, Stephan W. 2008. Population genetics of speciation in two closely related wild 787 tomatoes (Solanum section Lycopersicon). Genetics 178:339-350.

788 Subramanian L, et al. 2016. Centromere localization and function of Mis18 requires Yippee-like 789 domain-mediated oligomerization. EMBO Rep. 17:496-507.

790 Szklarczyk D, et al. 2017. The STRING database in 2017: quality-controlled protein-protein association 791 networks, made broadly accessible. Nucleic Acids Res. 45:D362-D368.

792 Tellier A, et al. 2011. Fitness effects of derived deleterious mutations in four closely related wild tomato $793 \quad$ species with spatial structure. Heredity 107:189-199.

794 The Tomato Genome Consortium. 2012. The tomato genome sequence provides insights into fleshy fruit 795 evolution. Nature 485:635-641.

796 Trapnell C, et al. 2012. Differential gene and transcript expression analysis of RNA-seq experiments 797 with TopHat and Cufflinks. Nat Protoc. 7:562-578.

$798 \mathrm{Vu}$ TM, et al. 2013. RNA-directed DNA methylation regulates parental genomic imprinting at several 799 loci in Arabidopsis. Development 140:2953-2960.

800 Walia H, et al. 2009. Dosage-dependent deregulation of an AGAMOUS-LIKE gene cluster contributes 801 to interspecific incompatibility. Curr Biol. 19:1128-1132.

802 Wolff P, Jiang H, Wang G, Santos-González J, Köhler C. 2015. Paternally expressed imprinted genes 803 establish postzygotic hybridization barriers in Arabidopsis thaliana. eLife 4:e10074.

804 Xie Z, Kasschau KD, Carrington JC. 2003. Negative feedback regulation of Dicer-Like1 in Arabidopsis 805 by microRNA-guided mRNA degradation. Curr Biol. 13:784-789.

806 Zhou M, Palanca AMS, Law JA. 2018. Locus-specific control of the de novo DNA methylation pathway 807 in Arabidopsis by the CLASSY family. Nat Genet. 50:865-873.

808 